\title{
Erratum: A three-dimensional engineered tumour for spatial snapshot analysis of cell metabolism and phenotype in hypoxic gradients
}

Darren Rodenhizer, Edoardo Gaude, Dan Cojocari, Radhakrishnan Mahadevan, Christian Frezza, Bradly G. Wouters and Alison P. McGuigan

Nature Materials http://dx.doi.org/10.1038/nmat4482 (2015); published online 23 November 2015; corrected online 1 December 2015.

In the version of the Article originally published online, in Fig. 1 there were some image display errors in panels $\mathbf{a}$ and $\mathbf{b}$ and the label 'GFP SK-OV-3' should have been green in panels c and e. In Fig. 2, panels a and b, layer numbers should have been defined. These errors have now been corrected in all versions of the Article. 